\title{
Intracavity mode competition between classes of flat-top beams
}

\author{
Igor Litvin ${ }^{\mathrm{a}, \mathrm{b}}$, Philip W. Loveday ${ }^{\mathrm{c}}$, Craig S. Long ${ }^{\mathrm{c}}$, Nikolai S. Kazak ${ }^{\mathrm{d}}$, Vladimir Belyi ${ }^{\mathrm{d}}$ and Andrew \\ Forbes ${ }^{\mathrm{a}, \mathrm{e} \dagger}$ \\ ${ }^{a}$ CSIR National Laser Centre, Box 395, Pretoria 0001, South Africa \\ ${ }^{b}$ Laser Research Institute, University of Stellenbosch, Stellenbosch 7602, South Africa \\ 'Sensor Science \& Technology, CSIR Material Science and Manufacturing, Box 395, Pretoria 0001, \\ South Africa \\ ${ }^{\mathrm{d}}$ B.I. Stepanov Institute of Physics, National Academy of Sciences of Belarus, 68 Nezavisimosti \\ ave., 220072 Minsk, Belarus

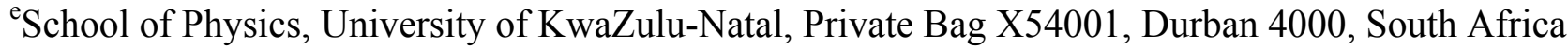

\begin{abstract}
There are many applications in which a laser beam with a flat-top intensity profile would be ideal, as compared to a laser beam with a non-uniform energy distribution. Standard stable optical resonators will unfortunately not generate such a laser beam as the oscillating mode. Single-mode oscillation would typically be Gaussian in profile, while multimode oscillation might deliver a beam with an averaged flat-like profile in the near field, but would diverge very quickly due to the higher order modes. In addition, if the modes are coherently coupled, then large intensity oscillations could be expected across the beam. Techniques exist to generate flat-top beams external to the cavity, but this is usually at the expense of energy, and almost always requires very precise input beam parameters. In this paper we present the design of an optical resonator that produces as the stable transverse mode a flat-top laser beam, by making use of an intra-cavity diffractive mirror. We consider the modal build-up in such a resonator and compare the mode competition between flattop like beams, including Flattened Gaussian beams, Fermi-Dirac beams, and super-Gaussian beams. Finally, we remark on the use of an intra-cavity piezoelectric unimorph mirror for selecting a particular class of flat-top beam as the fundamental mode of the resonator.
\end{abstract}

Keywords: Flat-top beams, resonator modes, diffractive optics, adaptive optics, piezoelectric unimorph mirror

\section{INTRODUCTION}

There are many applications in which a laser beam with a flat-top intensity profile would be ideal, as compared to a laser beam with a non-uniform energy distribution. Standard stable optical resonators will unfortunately not generate such a laser beam as the oscillating mode. Single-mode oscillation would typically be Gaussian in profile, while multimode oscillation might deliver a beam with an averaged flat-like profile in the near field, but would diverge very quickly due to the higher order modes. In addition, if the modes are coherently coupled, then large intensity oscillations could be expected across the beam. Techniques exist to generate flat-top beams external to the cavity, but this is usually at the expense of energy, and almost always requires very precise input beam parameters. A traditional laser resonator configuration is depicted in Fig. 1. It consists of a gain medium inside an optical cavity which is supplied with energy. Usually the cavity consists of two mirrors aligned such that the light passes through the gain medium several times, while traveling between the two mirrors. One of the two mirrors is made partially transparent, and the laser beam is emitted through this mirror (henceforth referred to as the output coupler).

† Corresponding author: Andrew Forbes; tel: +27 12841 2368; fax: +27 12841 3152; email: aforbes1@csir.co.za

Laser Beam Shaping IX, edited by Andrew Forbes, Todd E. Lizotte, Proc. of SPIE Vol. 7062,

706210, (2008) - 0277-786X/08/\$18 - doi: 10.1117/12.793686

Proc. of SPIE Vol. $7062706210-1$ 


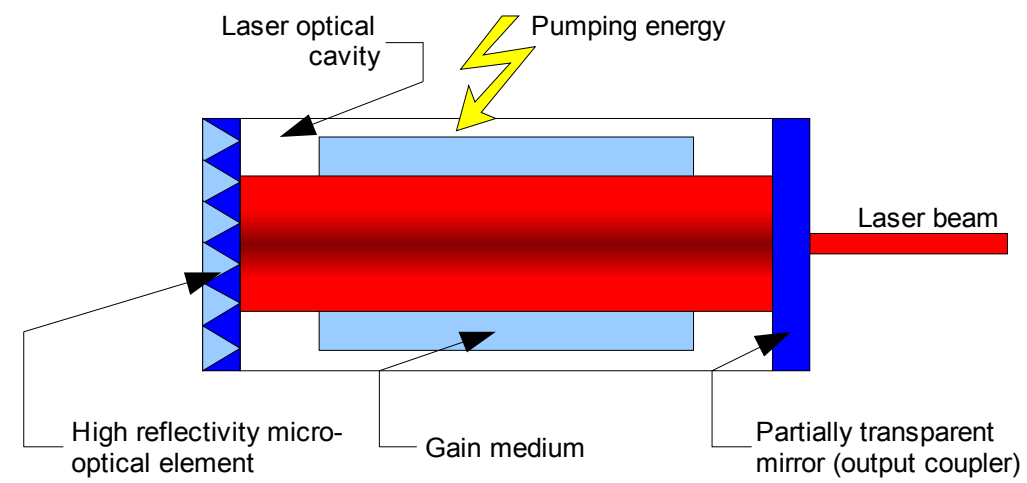

Fig. 1. Laser resonator with a gain medium and a diffractive micro-optical element on the left.

In the case depicted in Fig. 1 the back reflector, which would usually be a spherical curvature mirror, is shown as a nonconventional diffractive optical element (DOE). If the phase profile of this element is chosen so as to differ from spherical curvature, then it is possible to select transverse modes other than the conventional Helmhotz-Gauss and Laguerre-Gauss modes. Since the laser beam intensity and phase is selected inside the laser by the DOE, this is often referred to as intracavity laser beam shaping. While it is well known that transverse modes may be selected by amplitude means, even for very complex mode patterns ${ }^{1}$, this has disadvantages in that the round trip loss increases thus restricting such solutions to high gain lasers. Contrary to this, it has been shown that it is possible to use a DOE to select the resonant mode by means of phase rather than amplitude ${ }^{2,3}$, with the potential for greatly reduced losses in the medium. DOEs manipulate light by diffraction rather than reflection or refraction, and thus have feature sizes of the order of the wavelength of the light; this complicates fabrication and may lead to errors in the desired surface profile. If the surface structure of the element is of a kinoform nature (continuous relief surface), then the DOE acts as a phase-only element, without any additional diffraction losses.

\section{INTRACAVITY LASER BEAM SHAPING}

In this section we outline how the phase profile of the intracavity DOE is determined, and how this relates to the choice of the resonant mode inside the cavity.

\subsection{Resonator concept}

The method of using DOEs insider laser resonators was first proposed by Belanger and Pare, ${ }^{2,3}$, and which we outline briefly here for the convenience of the reader. Consider some arbitrary field that may be written in the form:

$$
u(x)=\psi(x) \exp [-i k \phi(x)],
$$

where $k=2 \pi / \lambda$ is the wavenumber of the laser beam, $\lambda$ is the wavelength, and $\psi(x)$ and $\phi(x)$ are the amplitude and phase of the electric field respectively. The action of a DOE in the form of a phase-only micro-optical mirror (as depicted in Fig. 1) is to transform the phase $\phi_{1}(x)$ of an incoming field to a new phase $\phi_{2}(x)$ of an outgoing field according to:

$$
\phi_{2}(x)=\phi_{1}(x)-2 \phi_{D O E}(x) .
$$

The salient point here is that this transformation takes place in a lossless manner, i.e., the outgoing amplitude $\psi_{2}(x)$ is unchanged. In particular, one can show ${ }^{2}$ that if the phase mirror is not spherical, then the change in phase also depends on the incoming field distribution $\psi_{1}(x)$. Thus it is expected that a phase-only mirror will discriminate against those modes that do not have the correct distribution $\psi_{1}(x)$. By invoking the requirement that the mode must reproduce itself after one round trip one can easily shown that the resulting restrictions on the phase of the DOE mirror is given by:

$$
\int_{-\infty}^{\infty} x\left(\frac{\partial \phi_{1}}{\partial x}\right) \psi_{1}^{2}(x) d x=\int_{-\infty}^{\infty} x\left(\frac{\partial \phi_{D O E}}{\partial x}\right) \psi_{1}^{2}(x) d x,
$$

from which we conclude that phase of the resonator eigenmode is the same as the phase of the DOE mirror, apart from a constant: 


$$
\phi_{D O E}(x)=\phi_{1}(x)-\phi_{1}(0) .
$$

Combining Eqs. (2) and (4), and ignoring the constant phase offset, we see that

$$
\phi_{2}(x)=-\phi_{1}(x) .
$$

Therefore the reflected beam $u_{2}(x)$ is the phase-conjugate of the incoming beam, $u_{2}(x)=u_{1}{ }^{*}(x)$. In this resonator only a particular beam distribution is phase conjugated by the DOE mirror, so that the eigenmode of the resonator satisfies the criteria that its wavefront matches the phase of each mirror in the cavity.

If we describe the desired field at the output coupler as $u_{O C}$, then reverse propagating the field to the DOE mirror using the Kirchhoff-Fresnel diffraction equation yields the field at the mirror as

$$
u_{D O E}(\rho, L)=-i^{n+1}(k / L) \exp (i k L) \exp \left(\frac{i k}{2 L} \rho^{2}\right) \int_{0}^{\infty} u_{O C}(r) J_{n}\left(\frac{k \rho r}{L}\right) \exp \left(\frac{i k}{2 L} r^{2}\right) r d r,
$$

where we have assumed that the resonator is rotationally symmetric and of optical path length $L$. If after refection off the DOE the field $u_{D O E}$ is to reproduce $u_{O C}$ at the output coupler, then the required phase for DOE mirror must be given by

$$
\phi_{D O E}=\operatorname{phase}\left[u_{D O E}^{*}(\rho, L)\right]
$$

with optical transfer function

$$
t_{D O E}=\frac{u_{D O E}^{*}}{u_{D O E}}
$$

This is the basis by which custom resonators may be designed. In addition, if one is able to deform the DOE mirror dynamically, then the optical transfer function in Eq. (8) may be controlled, thereby modifying the phase of the DOE and consequently the output field $u_{O C}$. Later we remark on how this may be achieved by using a piezoelectric unimorph mirror attached to the DOE mirror.

\subsection{Flat-top beams}

There are many classes of flat-top beams that exhibit very similar propagation properties ${ }^{4}$. It has already been shown previously that such beams are propagation invariant in a non-linearly absorbing medium, but diverge in their intensity profile very rapidly under free space propagation ${ }^{5,6}$. In many classes of flat-top beam, the rate of the divergence may be controlled by a scale parameter closely coupled to the steepness of the edges and the flatness of the intensity profile at the centre of the beam.

The exact definition of a flat-top beam (FTB) is one with constant intensity in some well defined radius, and zero intensity elsewhere:

$$
I_{F T B}(r)=\left\{\begin{array}{cc}
I_{0}, & r<w \\
0, & r>w
\end{array},\right.
$$

where $w$ is the radial width of the beam. There are several functions used to approximate this. The well-know superGaussian beam (SGB) has an intensity profile given by:

$$
I_{S G B}(r)=I_{0} \exp \left(-2(r / w)^{2 p}\right) .
$$

When the order parameter $p$ becomes large, $I_{S G B}(r)=I_{F T B}(r)$. In the special case that $p=1$, the intensity profile is the standard Gaussian. Closely related to the SGB are the Fermi-Dirac beam (FDB) and the Super-Lorentzian beam (SLB):

$$
I_{F D B}(r)=I_{0}\left(1+\exp \left[\beta\left(\left|\frac{r}{w}\right|-1\right)\right]\right)^{-1}
$$




$$
I_{S L B}(r)=I_{0}\left[1+\left(\frac{r}{w}\right)^{q}\right]^{-1}
$$

In Eqs. (11) and (12) the order parameters are $\beta$ and $q$ respectively, and have the same influence on the intensity profile as the order parameter $p$ in the case of the SGB. An often used distribution is that of the so-called Flattened Gaussian Beam (FGB) ${ }^{7}$ :

$$
u_{F G B}(r, z)=A \frac{w_{0 N}}{w_{N}(z)} \exp \left(i\left[k z-\Phi_{N}(z)\right]\right) \exp \left[\left(\frac{i k}{2 R_{N}(z)}-\frac{1}{w_{N}^{2}(z)}\right) r^{2}\right] \sum_{n=0}^{N} c_{n} L_{n}\left(\frac{2 r^{2}}{w_{N}^{2}(z)}\right) \exp \left[-2 i n \Phi_{N}(z)\right]
$$

Here the intensity of the beam is given by $I_{F G B}(r, z)=\left|u_{F G B}(r, z)\right|^{2}$ with beam parameters of new waist size, Rayleigh range, beam size, radius of curvature and Gouy phase shift given by:

$$
\begin{aligned}
& w_{0 N}=w_{0} / \sqrt{N} \\
& z_{r}=k w_{0 N}^{2} / 2 \\
& w_{N}(z)=w_{0 N} \sqrt{1+\left(z / z_{r}\right)^{2}} \\
& R(z)=z+z_{r}^{2} / z \\
& \Phi(z)=\tan ^{-1}\left(z / z_{r}\right)
\end{aligned}
$$

The summation is over a weighted set of Laguerre polynomials, where the weighting of factor $c_{n}$ of the $n$th Laguerre polynomial, $L_{n}$, is given by

$$
c_{n}=(-1)^{n} \sum_{m=n}^{N}\left(\begin{array}{l}
m \\
n
\end{array}\right) \frac{1}{2^{m}}
$$

The advantage of this profile over others is that Eq. (13) offers a simple analytical expression for its profile at any propagation distance $z$. Once again there is an order parameter associated with the field, given by the summation index $N$.

\section{NUMERICAL SIMULATIONS}

The question posed in this study is whether a DOE mirror designed for one class of flat-top beam can discriminate against modes described by the other classes. The designed axisymmetric DOE mirror is shown in Fig. 2(a), with associate output intensity given by Fig. 2(b). The element was designed for a $\mathrm{CO}_{2}$ laser cavity of length $L=1.772 \mathrm{~m}$, operating at a wavelength of $\lambda=10.6 \mu \mathrm{m}$. The DOE was designed so that a circular FGB of order $N=10$ is generated at the output coupler, with $w_{0}=10 \mathrm{~mm}$; the phase at the output coupler was set to 'flat', i.e., $R(0)=\infty$, with the DOE mirror placed well within the Rayleigh range of the beam, $L / z_{r} \sim 0.6$. Note that the surface height is modulated every $10.6 \mu \mathrm{m}$ in height, or one complete wavelength, corresponding to a $2 \pi$ phase shift of the light. An intracavity aperture was added to the resonator in the form of a $15 \mathrm{~mm}$ radius circular aperture placed at the DOE mirror in order to mimic the limited spatial extent of the electrodes of the $\mathrm{CO}_{2}$ laser.

Calculations of the mode were performed using the Fox-Li method ${ }^{8}$, starting from spontaneous emission (background noise). The field at the output coupler was computed for each complete round trip, and the losses for the $i$ th round trip were calculated as:

$$
\text { Loss }=1-\frac{\gamma_{i}}{\gamma_{i-1}}
$$

where $\gamma$ is the energy contained in the field. 


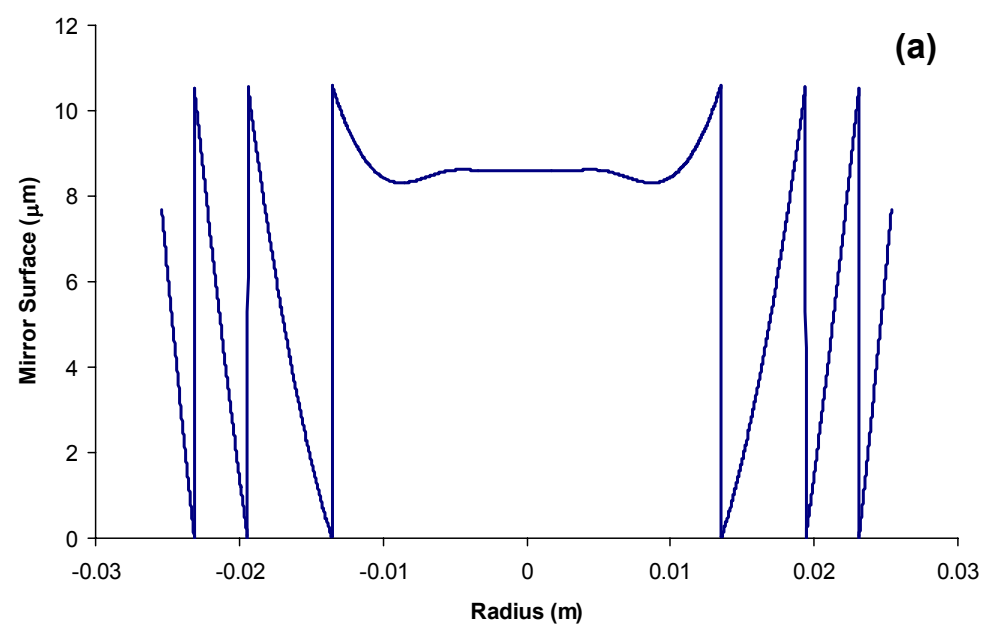

(b)

Fig. 2. Example of a DOE for outputting a FGB of order $N=10$ : (a) DOE mirror surface, and (b) contour plot of the expected beam intensity at the output coupler, showing a uniform intensity central region and steep edges.

\subsection{Modal build-up of the designed beam}

The modal build-up was computed starting from a background noise signal as shown in Fig. 3.

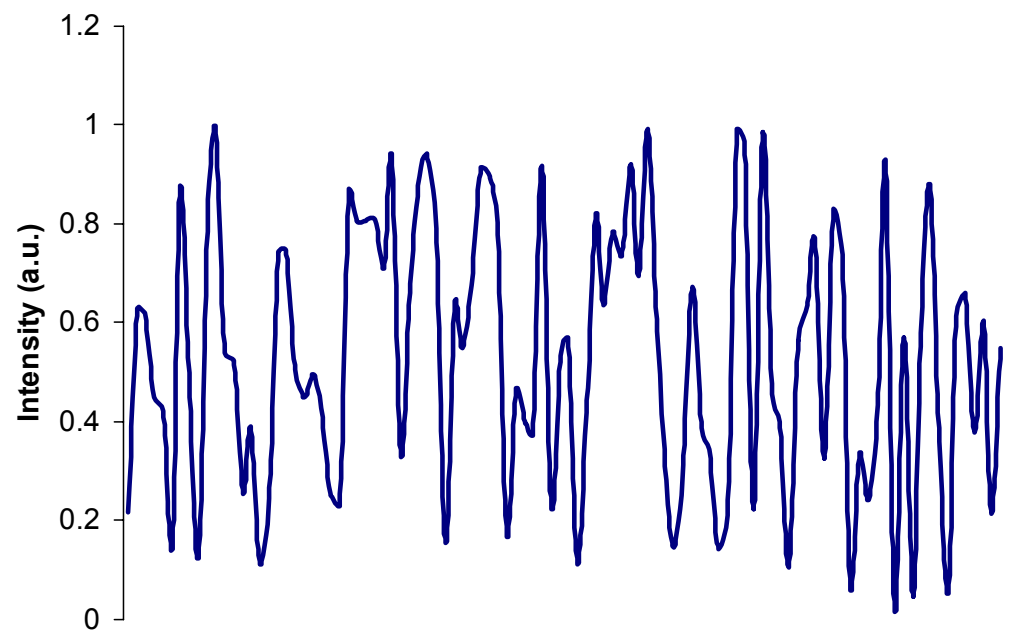

Fig. 3. Intensity of the oscillating field at the output coupler, starting from spontaneous emission (noise).

After approximately 250 round trips the losses of the field stabilized, indicating that a stable mode had formed. The final loss of the stable mode per round trip was found to be $0.2 \%$, which is close the theoretically predicted $0 \%$ loss for this conjugating resonator. 


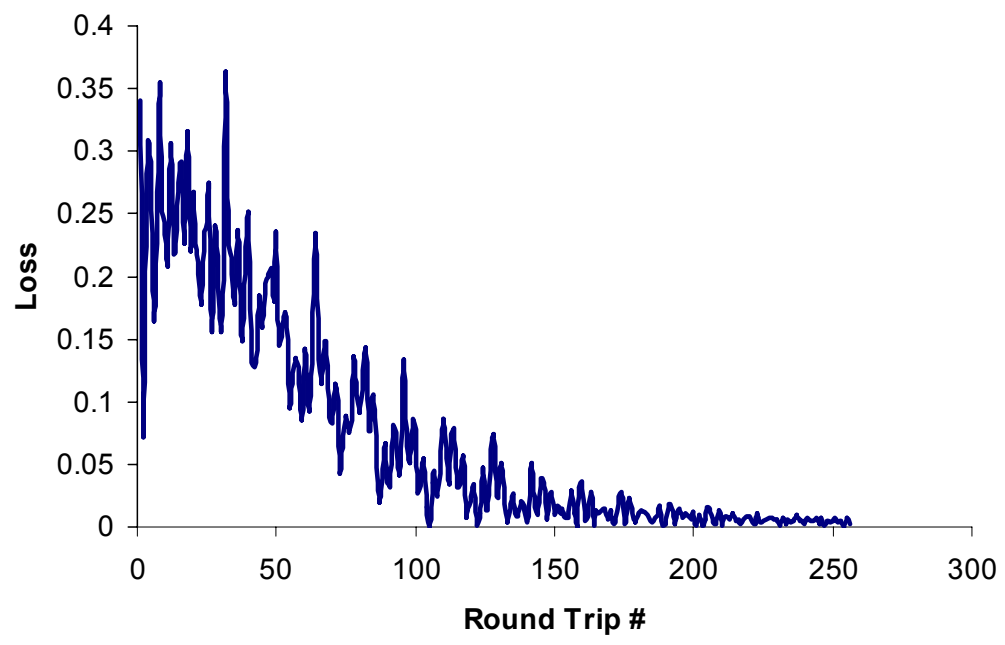

Fig. 4. Round trip loss starting from noise (high loss) finally converging to low loss after $\sim 250$ round trips.

At convergence, the wavefronts on both mirrors match that of the oscillating beam, as expected. The phase of the beam at the output coupler is shown in Fig. 5(a) and is clearly the desired 'flat' wavefront. Also shown in Fig. 5(b) is the intensity profile of the stable mode, showing a very good approximation to the desired flat-top beam. The fact that the losses are very low suggests that this resonator may be used for low gain laser systems. A time averaged field - what one would expect to measure experimentally - is also 'identical' to that shown in Fig. 5(b).
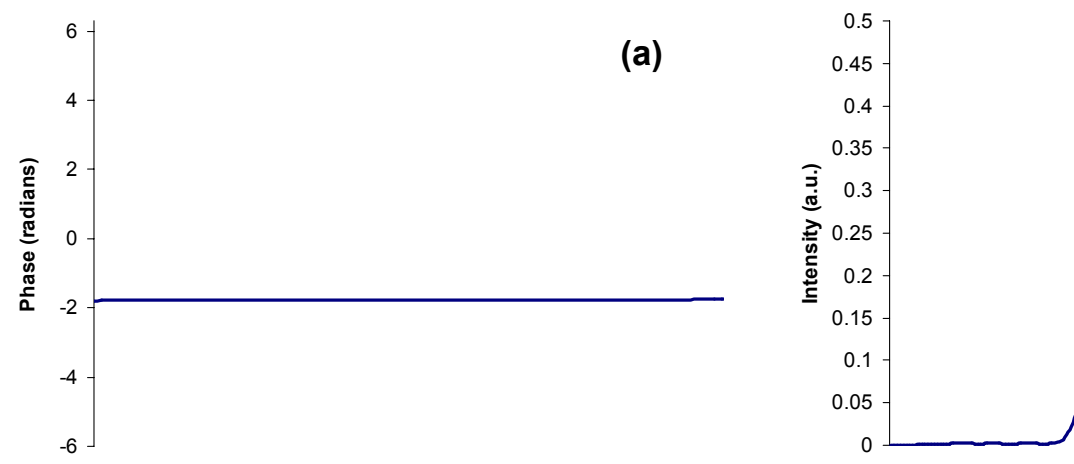

(b)

Fig. 5. (a) Phase of the oscillating mode at the output coupler, showing a flat wavefront, and (b) stable mode output intensity from the resonator.

After approximately 50 complete round trips the losses per round trip appear to enter into a brief oscillating cycle before the final low loss mode appears. A zoomed section of Fig. 4 is shown in Fig. 6. This periodicity may be understood by considering the DOE mirror's 'best fit' curvature and then modeling the resonator using an ABCD matrix approach. From this the eigenvalues and eigenvectors of a ray traversing the resonator may be found, and thus the periodicity for true repeatability. From this analysis we find that the resonator has a periodicity of $\sim 7.4$, which equates to either 15 ( 7.5 $\times 2)$ or $22(7.33 \times 3)$ complete passes (where one pass is from the output coupler to the DOE and back), which is consistent with the period of the oscillations uncovered with the Fox-Li analysis of $\sim 15$ complete passes. 


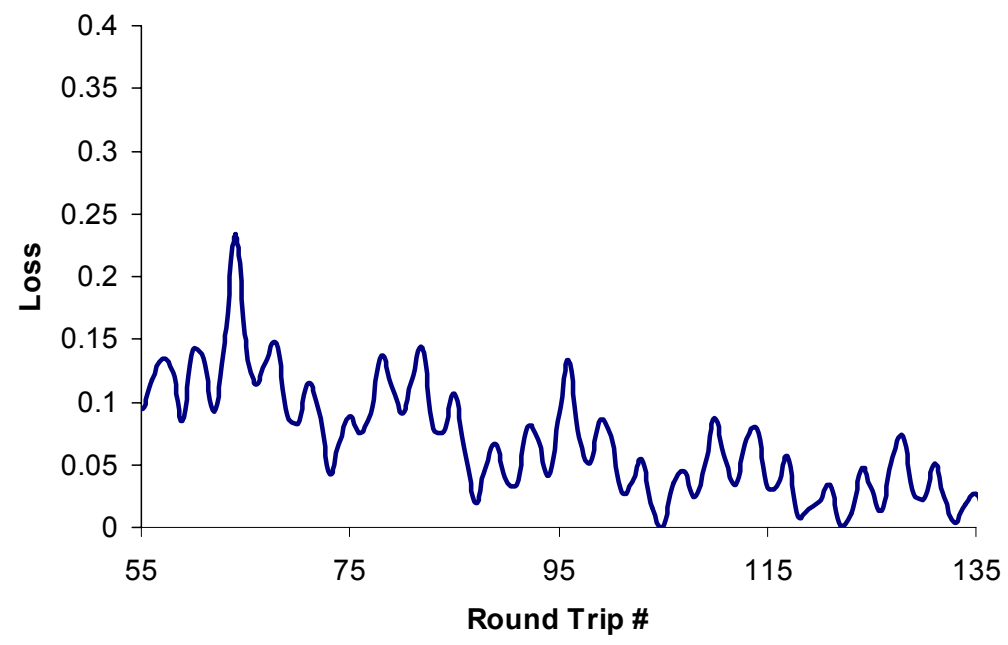

Fig. 6. The losses enter into an oscillating cycle once competing modes have had enough time to appear, but prior to the lowest loss mode dominating.

\subsection{Mode competition}

From the previous section we noted that mode competition is an important aspect of such resonators. In Fig. 7 we show the results of inserting other classes of flat-top beams into the resonator to determine their losses. In each case a flat-top beam was created using Eqs. (10)-(12), and inserted with a flat wavefront at the output coupler. Each field was propagated through one complete round trip and the losses calculated. Unsurprisingly, the losses increase as the order parameter is detuned away from the design beam order. However, as expected one can not easily distinguish between these classes of beams when their order is selected such that the intensity profile is similar to that of a FGB of $N=10$. However, the ability of the resonator to discriminate against those modes not matching this intensity profile is very evident, with losses that are orders of magnitude higher when the order parameter is detuned.

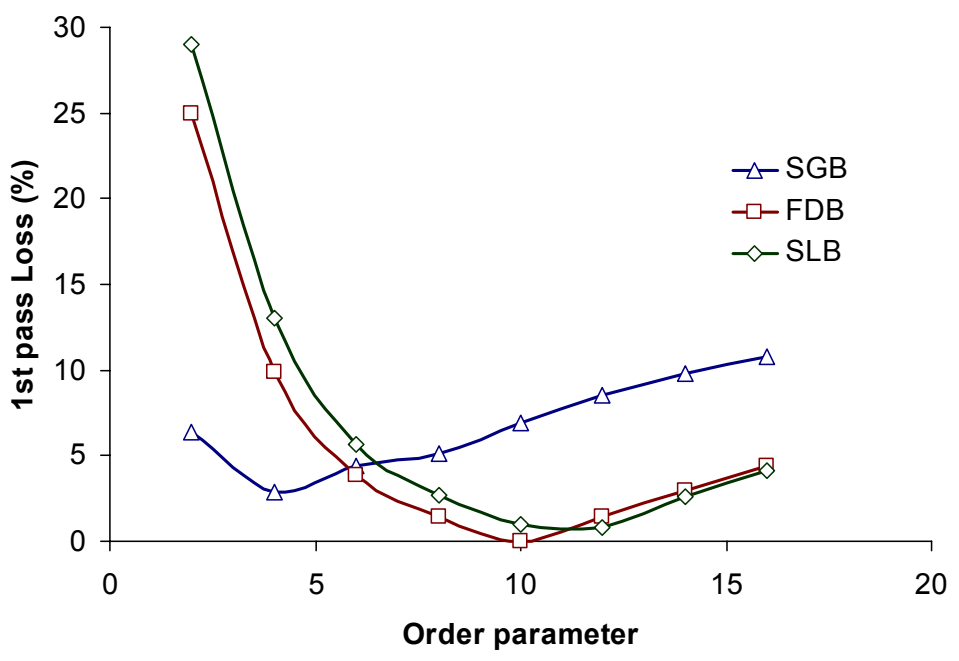

Fig. 7. One round trip loss as a function of the order parameter for three classes of flat-top beams similar to the designed FGB.

However, a small error in the phase of the mirror has a significant influence on the output mode from the resonator. Fig. 8 shows the resulting output intensity and phase at the output coupler when a small change is introduced to the DOE 
mirror. In this case, the central region of the mirror is too low by $\sim 0.2 \mu \mathrm{m}$, while the maximum error is approximately 6 $\mu \mathrm{m}$ in total.
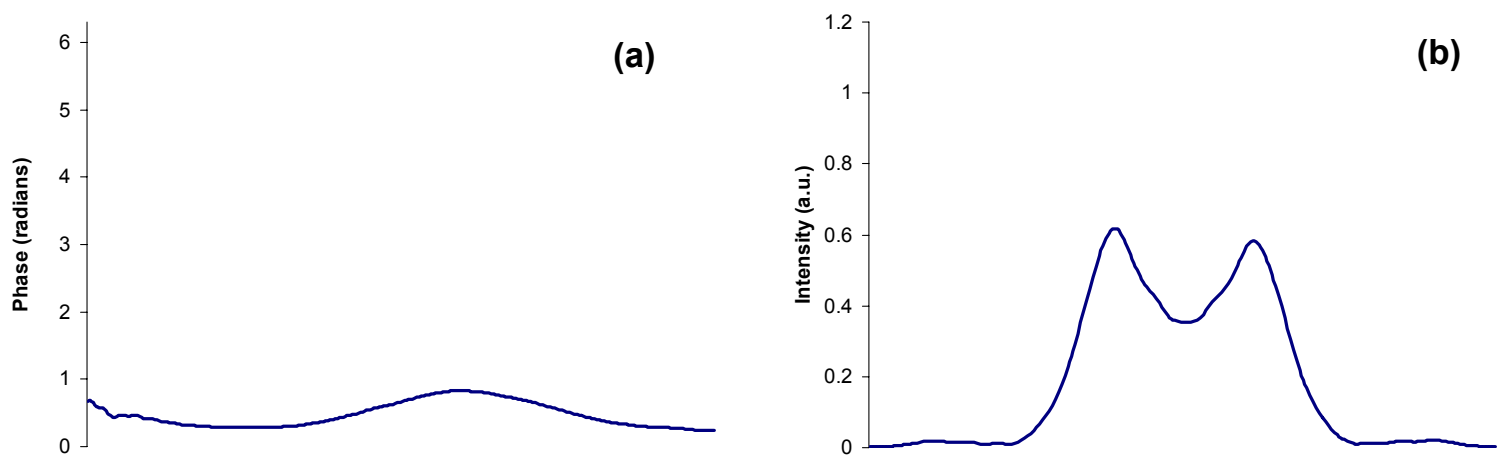

Fig. 8. (a) Phase of the oscillating mode at the output coupler, showing a slight deviation from a flat wavefront, and (b) the stable mode output intensity from the resonator with a large deviation from a flat-top beam.

From this it is clear that fabrication errors are an important likely contributor in determining the stable mode of the cavity. We therefore propose a DOE mirror with a static phase that can be adjusted with the aid of a deformable mirror, as outlined in the next section.

\section{MODE SELECTION WITH A DEFORMABLE MIRROR}

The mode that is achieved is influenced by the profile of the DOE and relatively small changes to this profile could be used to promote the formation of different beams. One method of adapting the profile of the DOE would be to integrate the DOE with a deformable mirror. A piezoelectric unimorph mirror is being investigated for this task. The device consists of a metallic disc, with the reflective surface, bonded to a piezoelectric disc, providing a small, low cost deformable mirror. Numerical modeling of such a device has been performed to estimate the deformations that can be achieved and this modeling has been verified by measurements on a prototype device ${ }^{9}$. The prototype and a measured displacement distribution are shown in Fig. 9. The prototype device had a diameter of $44 \mathrm{~mm}$ and produced a maximum displacement of approximately $40 \mu \mathrm{m}$. The electrode pattern, on the free surface of the piezoelectric disc, can be optimized to achieve a required deformation distribution of the mirror ${ }^{10}$. While it appears that sufficient deformations can be obtained the practical challenge of obtaining a flat mirror and then integrating this with the DOE remains.
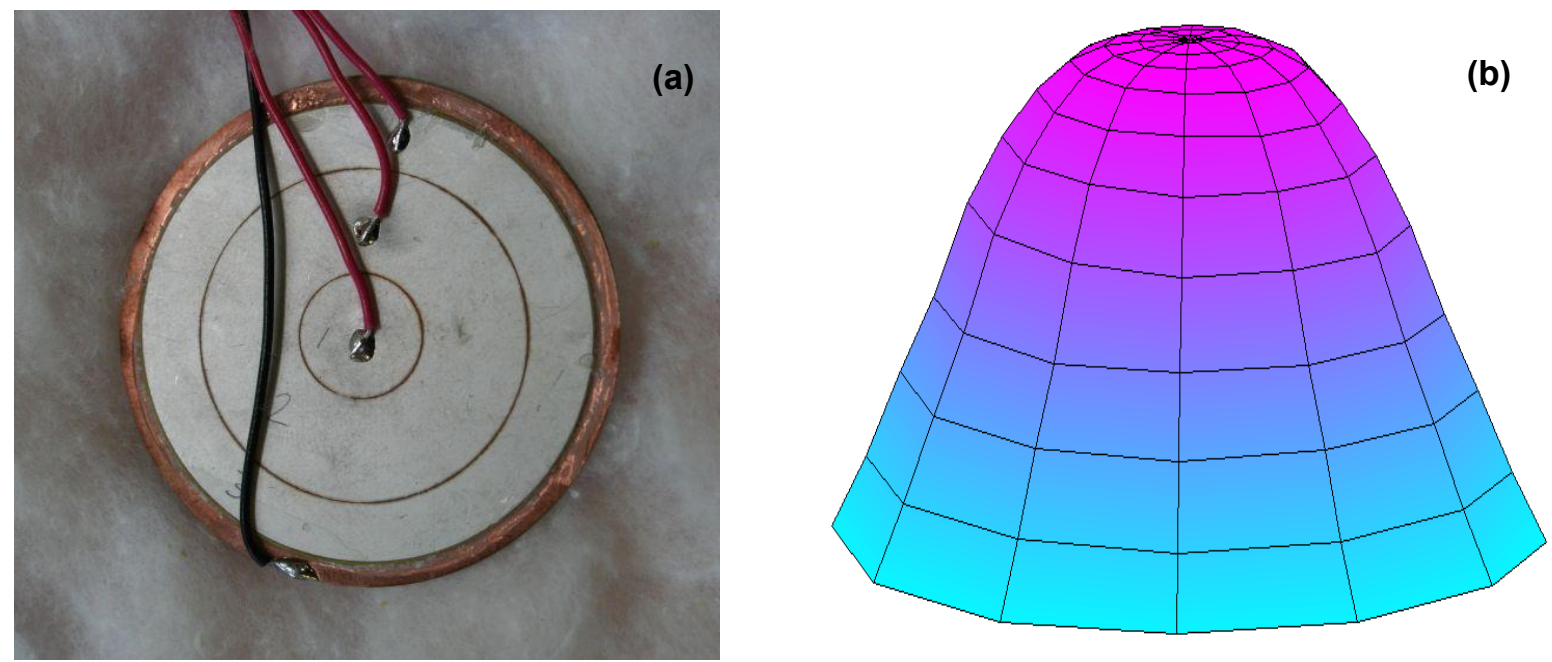

Fig. 9. (a) Rear view of prototype deformable mirror, and (b) typical measured displacement distribution. 


\section{CONCLUSION}

We have outlined the design approach for an optical resonator that produces as the stable transverse mode a flat-top laser beam, by making use of an intra-cavity phase-only diffractive mirror as the mode selective device. We considered the modal build-up in such a resonator and compared the mode competition between flat-top like beams, including Flattened Gaussian beams, Fermi-Dirac beams, and super-Gaussian beams. It would appear that the resonator is not able to distinguish these functions when the order parameters are shown such that they have similar intensities, but that there is large mode discrimination for orders that depart from the designed value. Finally, we remarked on the use of an intra-cavity piezoelectric unimorph mirror for selecting a particular class of flat-top beam as the fundamental mode of the resonator.

\section{REFERENCES}

[1] Litvin I.A., and Forbes A., "Bessel-Gauss resonator with internal amplitude filter," Opt. Commun., 281, 2385-2392 (2008).

[2] Belanger P.A., and Pare C., "Optical resonators using graded-phase mirrors," Optics Letters, 16(14), 1057-1059 (1991).

[3] Pare C. and Belanger P.A., "Custom laser resonators using graded-phase mirrors," IEEE Journal of Quantum Electronics, 28(1), 355-362 (1992).

[4] Shealy D.L., and Hoffnagle J.A., "Laser beam shaping profiles and propagation," Applied Optics, 45(21), 51185131 (2006).

[5] Forbes A., Strydom H.J., Botha L.R., and Ronander E., "Beam delivery for stable isotope separation," Proc. SPIE 4770, 13-27 (2002).

[6] Forbes A., "Laser beam propagation in non-linearly absorbing media," Proc. SPIE 6290, 629003 (2006).

[7] Gori F., "Flattened gaussian beams," Opt. Commun., 107, 335-341 (1994).

[8] Fox A.G., Li T., "Resonant modes in a maser interferometer," Bell Syst. Tech. J., 40, 453-488 (1961).

[9] Long C.S., Loveday P.W. and Forbes A., "A piezoelectric deformable mirror for intra-cavity laser adaptive optics", Smart Structures and Materials 2008: Industrial and Commercial Applications of Smart Structures Technologies, Proc. SPIE 6930, 69300Y-1 (2008).

[10] Long C.S., Loveday P.W. and Forbes A., "Development of a piezoelectric adaptive mirror for laser beam control", $11^{\text {th }}$ International Conference of New Actuators, Bremen, Germany, 9-11 June (2008). 